\title{
An Algorithm for Finding Large Induced Planar Subgraphs
}

\author{
Keith Edwards ${ }^{1}$ and Graham Farr $^{2, \star}$ \\ 1 Department of Applied Computing \\ University of Dundee \\ Dundee, DD1 4HN \\ U.K. \\ kedwards@computing.dundee.ac.uk \\ 2 School of Computer Science and Software Engineering \\ Monash University (Clayton Campus) \\ Clayton \\ Victoria 3168 \\ Australia \\ gfarr@csse.monash.edu.au
}

\begin{abstract}
This paper presents an efficient algorithm that finds an induced planar subgraph of at least $3 n /(d+1)$ vertices in a graph of $n$ vertices and maximum degree $d$. This bound is sharp for $d=3$, in the sense that if $\varepsilon>3 / 4$ then there are graphs of maximum degree 3 with no induced planar subgraph of at least $\varepsilon n$ vertices. Our performance ratios appear to be the best known for small $d$. For example, when $d=3$, our performance ratio of at least $3 / 4$ compares with the ratio $1 / 2$ obtained by Halldórsson and Lau. Our algorithm builds up an induced planar subgraph by iteratively adding a new vertex to it, or swapping a vertex in it with one outside it, in such a way that the procedure is guaranteed to stop, and so as to preserve certain properties that allow its performance to be analysed. This work is related to the authors' work on fragmentability of graphs.
\end{abstract}

\section{Introduction}

Finding a large planar subgraph of a graph is an important problem in graph drawing [5]. In this paper, an induced planar subgraphs is sought, and its size is taken to be the number of its vertices.

Formally, the problem we would like to solve is the following.

MAXIMUM INDUCED PLANAR SUBGRAPH (MIPS)

Input: Graph $G$, on $n$ vertices.

Output: A largest set of vertices $P \subseteq V(G)$ such that the induced subgraph $\langle P\rangle$ is planar.

Of course, one can also look at the complementary, and computationally equivalent, problem of finding the smallest set $R$ of vertices whose removal leaves

\footnotetext{
* Some of the work of this paper was done while the second author visited the Department of Applied Computing, University of Dundee, in July-September 2000.
}

P. Mutzel, M. Jünger, and S. Leipert (Eds.): GD 2001, LNCS 2265, pp. 75 83, 2002.

(C) Springer-Verlag Berlin Heidelberg 2002 
behind a planar graph (i.e., so that $\langle V(G) \backslash R\rangle$ is planar), and we sometimes find this point of view convenient.

Unfortunately, MIPS is NP-hard [1819, and is also hard to approximate 22]. Halldórsson [11 gives an approximation algorithm that finds an induced planar subgraph of size $\Omega\left(n^{-1}(\log n / \log \log n)^{2}\right)$ times the optimum. For graphs of maximum degree $d$, Halldórsson and Lau [12] give a linear time algorithm with a performance ratio of $1 /\lceil(d+1) / 3\rceil$.

In this paper, we present a polynomial time algorithm that finds an induced planar subgraph of at least $3 n /(d+1)$ vertices in a graph of maximum degree $d$. This bound is sharp for $d=3$, and some upper bounds are given for $d>3$. We also ask: for each $d$, what is the largest $\alpha(d)$ such that an induced planar subgraph of $\alpha(d) \cdot n$ vertices can be found in every graph of maximum degree $d$ ? Note that this ratio compares the size of the induced planar subgraph found with that of the whole graph, and so is not a performance ratio, though it certainly implies a performance ratio of at least $3 /(d+1)$. In a sense, our result removes the ceiling from the performance ratio of Halldórsson and Lau given above, which for graphs of low $d$ with $d \not \equiv 2 \quad(\bmod 3)$ is a significant improvement. For example, when $d=3$, our algorithm has performance ratio at least $3 / 4$, whereas that of 12 is $1 / 2$. Also, the induced planar subgraphs found by Halldórsson and Lau's algorithm have maximum degree at most 2 , whereas those found by our algorithm are not so restricted in structure.

The algorithm we present is virtually implicit in our proof of 8 , Theorem $3.2]$, though that proof is, on the face of it, non-algorithmic. Our purpose here is to state the algorithm clearly and explicitly to the graph drawing community, and to discuss its properties and some of the questions it raises.

The fact that the algorithm performs well for graphs of bounded degree suggests that it may be useful in practical applications, where graphs are often sparse (although that is not quite the same thing, of course).

We recognise that MIPS has attracted much less attention than the Maximum Planar Subgraph (MPS) problem, in which a planar subgraph in the usual sense (i.e., not necessarily induced) is sought, and its size is taken to be the number of its edges (and its vertex set may as well be the vertex set of the input graph); see, e.g., [15]16]17/20|23]. MPS is known to be NP-hard [21], even for graphs of maximum degree 3 9]. A number of exact or approximate algorithms have been developed and studied [2]3/4,7,10,15]16,23], and the best known performance ratio is 4/9, due to Călinescu et al. [3]. The literature on MIPS is much smaller. (Liebers [20] reviews MIPS in two pages, while devoting ten to MPS.) Apart from the complexity references cited above, we note work on finding large induced planar subgraphs of graphs of a given genus 61314.

Throughout the paper, $n$ is the number of vertices in the $\operatorname{graph}(\mathrm{s})$ under discussion, $m$ is the number of edges, and $d$ is the maximum degree. If $X \subseteq V(G)$ then $k(X)$ denotes the number of components of $\langle X\rangle$. If $X, Y \subseteq V(G)$, then $E(X, Y)$ denotes the set of edges with one endpoint in $X$ and the other in $Y$. If $v \in V(G)$ and $X \subseteq V(G)$, then $N_{X}(v)$ denotes the set of those vertices in $X$ that are adjacent to $v$. 


\section{The Algorithm}

By way of introduction, consider the following easy algorithm:

Algorithm 1. An obvious iterative algorithm

1. Input: Graph $G$.

2. $P:=\emptyset$

$R:=V(G)$

3. while ( there exists $x_{0} \in R$ such that

\{

$\left\langle P \cup\left\{x_{0}\right\}\right\rangle$ is planar )

$P:=P \cup\left\{x_{0}\right\}$

\}

$R:=R \backslash\left\{x_{0}\right\}$

This algorithm just keeps on adding vertices to $P$ for as long as it is possible to do so, while preserving planarity of $\langle P\rangle$. It is discussed briefly by Liebers [20], who notes that it has complexity $O(n m)$, where $m=|E(G)|$. Observe that, when it stops, each vertex in $R$ must be adjacent to at least two vertices in $P$ (else such a vertex could be added to $P$ by the algorithm). A simple counting argument then shows that the induced planar subgraph so found has at least $2 n /(d+2)$ vertices. (Consider $|E(P, R)|$. Count (or rather, bound) it in two ways.) Note that the same lower bound is achieved by an algorithm which replaces the condition in Step 3 of Algorithm 1 by the simpler test

( there exists $x_{0} \in R$ such that $\left.\operatorname{deg}_{P}\left(x_{0}\right) \leq 1 \quad\right)$

The modified algorithm may often do worse than Algorithm 1, and the vertex set $P$ found may not even be maximal (subject to planarity of $\langle P\rangle$ ). Nonetheless, the set $P$ found still has at least $2 n /(d+2)$ vertices. It will also be easier to implement, and faster.

The algorithm we now present improves on this basic iterative approach by examining more carefully whether the vertex under consideration, $x_{0}$, can usefully be added to $P$. Sometimes it is advantageous to swap $x_{0}$ with some vertex already in $P$. Care is needed to ensure that an algorithm that does this will eventually stop.

The algorithm is based on the proof of [8, Theorem 3.2].

The algorithm maintains four sets of vertices of the input graph $G: P$, the set of vertices of the induced planar subgraph constructed so far; $R=V(G) \backslash P$; $F$, the set of vertices that belong to those components of $\langle P\rangle$ that are trees (i.e., the $f$ orest part of $\langle P\rangle$ ); and $N=P \backslash F$ (non-trees).

Algorithm 2. Finding an induced planar subgraph of at least $3 n /(d+1)$ vertices.

1. Input: Graph $G$.

2. $P:=\emptyset$

$N:=\emptyset$

$F:=\emptyset$

$R:=V(G)$ 
3. while ( there exists $x_{0} \in R$ such that

$$
\left.N_{N}\left(x_{0}\right) \leq 1 \text { or }\left(N_{N}\left(x_{0}\right)=2 \text { and } N_{F}\left(x_{0}\right) \leq 1\right)\right)
$$

3.1. if $\left(N_{N}\left(x_{0}\right) \leq 1\right)$

$$
\begin{aligned}
& P:=P \cup\left\{x_{0}\right\} \\
& R:=R \backslash\left\{x_{0}\right\} \\
& \text { update } N, F .
\end{aligned}
$$

3.2. else if (the two vertices $y, z$ in $N_{N}\left(x_{0}\right)$ are in different components of $N$ )

$$
\begin{aligned}
& P:=P \cup\left\{x_{0}\right\} \\
& R:=R \backslash\left\{x_{0}\right\} \\
& \text { update } N, F .
\end{aligned}
$$

3.3. else if (there is a unique $y-z$ path in $\langle N\rangle$ )

$$
\begin{aligned}
& P:=P \cup\left\{x_{0}\right\} \\
& R:=R \backslash\left\{x_{0}\right\} \\
& \text { update } N, F .
\end{aligned}
$$

3.4. else

Let $N_{0}$ be the vertex set of the component of $N$ containing $y$ and $z$.

Find a $y-z$ path $\Pi$ in $\left\langle N_{0}\right\rangle$.

$$
\text { Let it be } y=x_{1}, x_{2}, \ldots, x_{t}=z \text {. }
$$

$x_{i}:=$ first vertex on $\Pi$ (when going from $y$ towards $z$ ) such that: there is a path $\Pi^{\prime}$ in $\left\langle N_{0}\right\rangle$ from $x_{i}$ to some later $x_{j}, j>i$, with $\Pi^{\prime}$ disjoint from $\Pi$ except at its endpoints.

$P:=\left(P \backslash\left\{x_{i}\right\}\right) \cup\left\{x_{0}\right\}$

$R:=\left(R \backslash\left\{x_{0}\right\}\right) \cup\left\{x_{i}\right\}$

update $N, F$.

4. Output: $P$.

Theorem 1 Algorithm 2 finds an induced planar subgraph of at least $3 n /(d+1)$ vertices.

Proof. See [8] Theorem 3.2].

In outline, the proof proceeds as follows. In Algorithm 2, it is easy to see that conditions $3.1,3.2$ and 3.3, if satisfied, allow the vertex $x_{0}$ to be added to $P$. A little more effort shows that, whenever step 3.4 is performed, the graph resulting from this step is planar. Theorem 2 below shows that the algorithm finds an induced planar subgraph. When the algorithm stops, each $v \in R$ satisfies either $d_{N}(v) \geq 3$ or $d_{N}(v)=2 \wedge d_{F}(v) \geq 2$. These together imply $d_{P}(v) \geq 3+d_{F}(v) / 2$. If $d \geq 4$, we count the edges of $E(P, R)$ in two ways (once from $P$, once from $R$ ) and derive (at some length) inequalities yielding the claimed bound. If $d=3$, count $E(N, R)$ instead. 


\section{Discussion}

\subsection{Complexity}

Theorem 2 Algorithm 2 has time complexity $O(\mathrm{~nm})$.

Proof. At each step, Algorithm 2 either

(i) increases $|P|$, while keeping $\langle P\rangle$ planar, or

(ii) decreases $|E(P)|$, while keeping $|P|$ unchanged and maintaining planarity of $\langle P\rangle$, or

(iii) decreases $k(P)$, while keeping $|P|$ and $|E(P)|$ unchanged and maintaining planarity of $\langle P\rangle$.

It follows that the algorithm stops eventually. In fact, if $s_{(i)}, s_{(i i)}, s_{(i i i)}$ denote the number of steps of Algorithm 2 that do (i), (ii) and (iii) respectively in the above list, and the total number of steps is $s=s_{(i)}+s_{(i i)}+s_{(i i i)}$, then we can find a linear bound on $s$ as follows. It can be shown that any step of type (i) increases $|E(P)|+k(P)$ by at most 2, and any step of type (ii) or (iii) decreases $|E(P)|+k(P)$ by exactly 1 . Hence

$$
2 s_{(i)}-s_{(i i)}-s_{(i i i)} \geq|E(P)|+k(P) .
$$

Combining this with the obvious $s_{(i)}=n$ gives $s_{(i i)}+s_{(i i i)} \leq 2 n$, so that $s \leq 3 n$. (In fact, using $|E(P)| \geq|P|-k(P)$ here gives $s \leq 3 n-|P|$. Using our bound on $|P|$ (Theorem 1), we obtain $s \leq 3 n d /(d+1)$.) The time taken by each step is no worse than linear in $m$, so the time complexity is $O(n m)$.

In the light of the superficial similarity of MIPS and MPS, it is interesting to note that Algorithm 2 actually tries (in steps of type (ii)) to decrease the number of edges in $\langle P\rangle$ in situations when it cannot increase $|P|$. Of course, this is quite understandable, as graphs with fewer edges should generally be more amenable to the addition of vertices.

\subsection{Performance}

It is natural to ask whether our lower bound of $3 n /(d+1)$ is the best possible, or whether there is some constant $\varepsilon>3 /(d+1)$ such that every graph on $n$ vertices with maximum degree $d$ has an induced planar subgraph of at least $\varepsilon n$ vertices. Define $\alpha(d)$ by

$$
\begin{aligned}
\alpha(d)=\sup \{\varepsilon \mid & \text { every } G \text { such that } \Delta(G) \leq d \\
& \text { has an induced planar subgraph of } \geq \varepsilon n \text { vertices }\} .
\end{aligned}
$$

We ask, then, for the value of $\alpha(d)$. Theorem 1 shows that

$$
\alpha(d) \geq 3 /(d+1) .
$$

On the other hand, the largest induced planar subgraph of $K_{d+1}$ is $K_{4}$, so

$$
\alpha(d) \leq 4 /(d+1) .
$$


Furthermore, the result [8, Theorem 3.3] implies that

$$
\alpha(d) \leq d /(2 d-2)
$$

This upper bound is better than (2) for $d \leq 5$, and together with (11) it gives us the exact value

$$
\alpha(3)=3 / 4 \text {. }
$$

Bühler [1] has calculated the minimum size of all maximum induced planar subgraphs of $d$-regular graphs on $n$ vertices, for several values of $n$ and $d$. This is the quantity

$$
\operatorname{mips}(n, d)=\min \{\operatorname{mips}(G) \mid G \text { is } d \text {-regular and }|V(G)|=n\},
$$

where $\operatorname{mips}(G)$ denotes the size of the maximum induced planar subgraph of $G$. We give her results in the right column of the following table, with our lower bound $3 n /(d+1)$ in the third column for comparison. Clearly our bound is not tight, though whether the difference between it and $\operatorname{mips}(n, d)$ is just $o(n)$, or is $\Theta(n)$ and so $\alpha(d)>3 /(d+1)$, is not known to us.

\begin{tabular}{|c|c|c|c|}
\hline$d$ & $n$ & $\lceil 3 n /(d+1)\rceil$ & $\operatorname{mips}(n, d)$ by [1] \\
\hline \hline 3 & 10 & 8 & 8 \\
& 12 & 9 & 10 \\
& 14 & 11 & 11 \\
& 16 & 12 & 13 \\
\hline 4 & 10 & 6 & 8 \\
& 11 & 7 & 9 \\
& 12 & 8 & 9 \\
& 13 & 8 & 10 \\
\hline 5 & 12 & 6 & 8 \\
\hline
\end{tabular}

\subsection{One More Step}

In some cases, it is possible to add one more vertex to $P$ after Algorithm 2 stops. This actually allows us to obtain a sharper bound on maximum induced planar subgraph size.

Theorem 3 If $G$ is a graph with $d=3, n \equiv 0$ or $1 \quad(\bmod 4)$ and, after Algorithm 2 stops, $|P|=\lceil 3 n / 4\rceil$, then any vertex $v \in R$ has the property that $\langle P \cup\{v\}\rangle$ is planar.

Proof. Suppose $d=3$. Observe first that the stopping condition of the algorithm implies that $R$ forms an independent set in $G$, and that each of its vertices have 3 neighbours in $P$. Furthermore, if $G$ is connected then $\langle P\rangle$ is too, else another step of type (i), (ii) or (iii) would be possible and the algorithm would not have stopped. 
Choose any $v \in R$, and put $P^{\prime}=P \cup\{v\}$ and $R^{\prime}=R \backslash\{v\}$. Observe that $\left|E\left(R^{\prime}\right)\right|=0$, and if $|P|=\lceil 3 n / 4\rceil$ then $\left|R^{\prime}\right|=\lfloor n / 4\rfloor-1$ so that $\left|E\left(P^{\prime}, R^{\prime}\right)\right|=$ $3\left|R^{\prime}\right|=3(\lfloor n / 4\rfloor-1)$. Hence we have

$$
\begin{aligned}
\left|E\left(P^{\prime}\right)\right| & =|E|-\left|E\left(P^{\prime}, R^{\prime}\right)\right|-\left|E\left(R^{\prime}\right)\right| \\
& \leq 3\lfloor n / 2\rfloor-3(\lfloor n / 4\rfloor-1) \\
& <\lceil 3 n / 4\rceil+4,
\end{aligned}
$$

provided $n \equiv 0$ or $1 \quad(\bmod 4)$ (for the sake of the final inequality). Now, if $\left\langle P^{\prime}\right\rangle$ were nonplanar, it would contain a subdivision of $K_{3,3}$. Since it is connected, we would have $\left|E\left(P^{\prime}\right)\right|=\left|P^{\prime}\right|+3 \geq\lceil 3 n / 4\rceil+4$, a contradiction. Hence $\left\langle P^{\prime}\right\rangle$ is planar.

So, when $d=3$ and $n \equiv 0$ or $1 \quad(\bmod 4)$, we can ensure that $|P| \geq\lceil 3 n / 4\rceil+$ 1: either Algorithm 2 gives such a $P$, or, if not, we can add any vertex into $P$ to achieve it. We can summarise the achievements of both Algorithm 2 and Theorem 3 by saying that, in combination, they give an induced planar subgraph of at least $\lceil(3 n+2) / 4\rceil$ vertices when $d=3$ : for all $G$ on $n$ vertices with $d=3$,

$$
\operatorname{mips}(G) \geq\lceil(3 n+2) / 4\rceil .
$$

It is intriguing to note that this refined lower bound equals Bühler's calculation of $\operatorname{mips}(n, 3)$ for all the values quoted for $d=3$ above. We find (using Bühler's program) that they are also equal for the odd values $n=9,11,13$, with the caveat that mips $(n, 3)$ is taken to be a minimum over graphs of maximum degree 3 since such $G$ cannot be 3 -regular. This raises the question of whether our refined lower bound is actually sharp, for $d=3$, in the much stronger sense of actually equalling $\operatorname{mips}(n, 3)$, and not just differing from it by $o(n)$ (which is what (3) tells us). This looks unlikely, however: a program of Bühler's was used to establish that mips $(18,3)=15$, whereas our lower bound is 14 .

\subsection{Modifications for Maximality}

As with the modified version of Algorithm 1, Algorithm 2 does not necessarily find a maximal induced planar subgraph. (Indeed, Theorem 3 gives cases where it never does.) It can easily be extended to do so, in various ways. The main loop control in Step 3 can be replaced by

$$
\begin{aligned}
\text { while }( & {\left[\exists x_{0} \in R:\left\langle P \cup\left\{x_{0}\right\}\right\rangle \text { is planar }\right] \text { or } } \\
{\left[\exists x_{0} \in R, x \in P:\right.} & \left(\left\langle(P \backslash\{x\}) \cup\left\{x_{0}\right\}\right\rangle \text { is planar }\right) \text { and } \\
& \left(\left[\left|E\left((P \backslash\{x\}) \cup\left\{x_{0}\right\}\right)\right|<|E(P)|\right]\right. \text { or } \\
& {\left[\left(\left|E\left((P \backslash\{x\}) \cup\left\{x_{0}\right\}\right)\right|=|E(P)|\right)\right. \text { and }} \\
& \left.\left.\left.\left.\left(k\left((P \backslash\{x\}) \cup\left\{x_{0}\right\}\right)<k(P)\right)\right]\right)\right]\right)
\end{aligned}
$$

The tests of Steps 3.1-3.3 could be combined and replaced by a single test of whether or not $\left\langle P \cup\left\{x_{0}\right\}\right\rangle$ is planar. The resulting algorithm will find a maximal induced planar subgraph, and should in practice produce larger induced planar subgraphs than Algorithm 2. On the other hand, it will be slower, harder to implement from scratch, and the techniques we have discussed here and in [8] do not give a stronger result on its performance. 


\section{References}

1. S. Bühler, Planarity of Graphs, M.Sc. dissertation, University of Dundee, 2000.

2. J. Cai and X. Han and R. E. Tarjan, An O $(m \log n)$-Time Algorithm for the Maximal Planar Subgraph Problem, SIAM J. Comput. 22 (1993) 1142-1162.

3. G. Călinescu, C. G. Fernandes, U. Finkler and H. Karloff, A better approximation algorithm for finding planar subgraphs, in: Proc. 7th Ann. ACM-SIAM Symp. on Discrete Algorithms (Atlanta, GA, 1996); also: J. Algorithms 27 (1998) 269-302.

4. Robert Cimikowski, An analysis of some heuristics for the maximum planar subgraph problem, in: Proc. 6th Ann. ACM-SIAM Symp. on Discrete Algorithms (San Francisco, CA, 1995), pp 322-331, ACM, New York, 1995.

5. G. Di Battista, Peter Eades, Roberto Tamassia and I. Tollis, Graph Drawing: Algorithms for the Visualization of Graphs, Prentice Hall, 1999.

6. H. N. Djidjev and S. M. Venkatesan, Planarization of graphs embedded on surfaces, in: M. Nagl (ed.), Proc. 21st Internat. Workshop on Graph-Theoretic Concepts in Computer Science (WG'95) (Aachen, 1995), Lecture Notes in Comput. Sci., 1017, Springer, Berlin, 1995, pp. 62-72.

7. M. E. Dyer, L. R. Foulds and A. M. Frieze, Analysis of heuristics for finding a maximum weight planar subgraph, Eur. J. Operational Res. 20 (1985) 102-114.

8. K. Edwards and G. Farr, Fragmentability of graphs, J. Combin. Theory (Ser. B) 82 (2001) 30-37.

9. L. Faria, C. M. H. de Figueiredo and C. F. X. Mendonça, Splitting number is NP-complete, in: Juraj Hromkovic and Ondrej Sýkora (eds.), Proc. 24th Internat. Workshop on Graph-Theoretic Concepts in Computer Science (WG '98) (Smolenice Castle, Slovakia, 18-20 June 1998), Lecture Notes in Comput. Sci., 1517, Springer, 1998, pp. 285-297.

10. O. Goldschmidt and A. Takvorian, An efficient graph planarization two-phase heuristic, Networks 24 (1994) 69-73.

11. Magnús M. Halldórsson, Approximation of weighted independent set and hereditary subset problems, Journal of Graph Algorithms and Applications 4 (1) (2000) $1-16$.

12. Magnús M. Halldórsson and Hoong Chuin Lau, Low-degree graph partitioning via local search with applications to constraint satisfaction, max cut, and coloring, Journal of Graph Algorithms and Applications 1 (3) (1997) 1-13.

13. J. P. Hutchinson, On genus-reducing and planarizing algorithms for embedded graphs, in: R. B. Richter (ed.), Graphs and Algorithms, Contemporary Mathematics 89, Amer. Math. Soc., Providence, RI, 1989.

14. Joan P. Hutchinson and Gary L. Miller, On deleting vertices to make a graph of positive genus planar, Discrete algorithms and complexity (Kyoto, 1986), Perspect. Comput., 15, pp. 81-98, Academic Press, Boston, MA, 1987.

15. M. Jünger and P. Mutzel, The polyhedral approach to the maximum planar subgraph problem: new chances for related problems, Graph Drawing '94, pp. 119-130.

16. M. Jünger and P. Mutzel, Maximum planar subgraphs and nice embeddings: practical layout tools, Algorithmica 16 (1996) 33-59.

17. P. C. Kainen, A generalization of the 5-color theorem, Proc. Amer. Math. Soc. 45 (1974) 450-453.

18. M. S. Krishnamoorthy and N. Deo, Node-deletion NP-complete problems, SIAM J. Comput. 8 (1979) 619-625.

19. J. M. Lewis and M. Yannakakis, The node-deletion problem for hereditary properties is NP-complete, J. Comput. System Sci. 20 (1980) 219-230. 
20. Annegret Liebers, Planarizing graphs - a survey and annotated bibliography, Journal of Graph Algorithms and Applications 5 (1) (2001) 1-74.

21. P. C. Liu and R. C. Geldmacher, On the deletion of nonplanar edges of a graph, Proc. 10th. S-E Conf. on Comb., Graph Theory, and Comp. 1977, Congressus Numerantium, No. 24 (1979) 727-738.

22. C. Lund and M. Yannakakis, The approximation of maximum subgraph problems, in: Proc. 20th Int. Colloquium on Automata, Languages and Programming (ICALP), Lecture Notes in Comput. Sci., 700, Springer-Verlag, 1993, pp. 40-51.

23. P. Mutzel, The Maximum Planar Subgraph Problem, Ph.D. Thesis, Univ. zu Köln, 1994. 